\title{
Uso de escamas y huesos de cachama blanca (Piaractus brachypomus) y tilapia (Oreochromis spp) como correctivo del suelo
}

\section{Use of scales and bones of white cachama (Piaractus brachypomus) and}

tilapia (Oreochromis spp.) as soil corrective

\author{
Perez Fajardo Esneyder Joany ${ }^{1}$, Arboleda Zapata Yurany Astrid ${ }^{1} y$ \\ Hurtado Nery Víctor Libardo ${ }^{2}$ \\ ${ }^{1}$ Ingenieros Agrónomos, Universidad de los Llanos y \\ ${ }^{2}$ Médico Veterinario Zootecnista, MSc, PhD, Docente Universidad de los Llanos \\ vhurtado@unillanos.edu.co
}

Recibido 04 de Julio 2017, Aceptado 19 de Octubre 2017

\section{RESUMEN}

La escama de pescado contiene elementos como el calcio, carbono y fósforo, que pueden aprovecharse con fines productivos agrícolas de importancia para la región de la Orinoquia colombiana, posibilitando el remplazo o la reducción de los productos químicos agrícolas que en la actualidad se utilizan para fertilizar el suelo, por lo tanto este trabajo se realizó con el objetivo de encontrar posibles alternativas de aprovechamiento de la escama y huesos de cachama blanca (Piaractus brachypomus) y tilapia roja (Oreochromis spp.), que se producen como desechos en restaurantes, hogares y piscícolas, y algunos de ellos los vierten al ambiente natural circunvecino, generando impactos ecológicos aún no evaluados. Se realizó la extracción de los componentes minerales y orgánicos presentes en la escama y los huesos, para posteriormente aplicarlo en el suelo de parcelas de un $\mathrm{m}^{2}$, establecidas en campo y con previo análisis químico del mismo para conocer su composición; adicionalmente se utilizó un producto comercial (cal dolomita) para comparar la eficiencia de las dos enmiendas. Se encontraron diferencias $(p<0.05)$ entre las dos fuentes de minerales, donde se destaca el incremento de fósforo en el suelo donde se ensayó la escama de pescado, cuyo nivel paso de 5.6 a 137 ppm, por lo tanto la escama de pescado podría ser utilizada como 
enmienda para suelos ácidos, aunque su efectividad no es igual a una enmienda comercial, su funcionalidad es óptima.

Palabras clave: Enmienda, abono, fertilizante, suelo acido, agricultura.

\begin{abstract}
The fish scale contains elements such as calcium, carbon and phosphorus, that can be used for agricultural productive purposes of importance to the region of the Colombian Orinoquia, enabling the replacement or reduction of agricultural chemicals that are currently used to fertilize the soil, therefore this work was done with the aim of finding possible alternative uses of the scales and bones of white cachama (Piaractus brachypomus) and red tilapia (Oreochromis spp.) that are produced as waste in restaurants, homes and fish farms, and some of them pour them into the surrounding natural environment, generating ecological impacts not yet evaluated. The extraction of the mineral and organic components present in the scale and bones was carried out, to later apply it on the floor of plots of one $\mathrm{m}^{2}$, established in the field and with previous chemical analysis of it to know its composition; in addition, a commercial product (dolomite lime) was used to compare the efficiency of the two amendments. Differences were found $(p<0.05)$ between the two mineral sources, where the increase in phosphorus in the soil where the fish scale was tested is highlighted, whose level went from 5.6 to 137 ppm, therefore the fish scale could be used as an amendment for acid soils, although its effectiveness is not equal to a commercial amendment, its functionality is optimal.
\end{abstract}

Keywords: Amendment, compost, fertilizer, acid soil, agriculture.

\title{
RESUMO
}

A escama de peixes contém elementos como cálcio, carbono e fósforo, que podem ser usado para fins produtivos agrícolas de importância para a região da Orinoquia colombiana, permitindo a substituição ou redução de produtos químicos agrícolas que atualmente são utilizados para fertilizar o solo, portanto, esse 
trabalho foi realizado para encontrar possíveis alternativas de uso das escamas e ossos de cachama branca (Piaractus brachypomus) e tilapia vermelha (Oreochromis spp.) que são produzidos como resíduos em restaurantes, casas e fazendas de peixes, e alguns deles os derramam no ambiente natural circundante, gerando impactos ecológicos ainda não avaliados. Foi realizada a extração dos componentes minerais e orgânicos presentes na escala e nos ossos, para depois aplicá-lo no chão de parcelas de um $\mathrm{m}^{2}$, estabelecido no campo e com análises químico do mesmo para conhecer sua composição; além disso, um produto comercial (cal dolomita) foi utilizado para comparar a eficiência das duas emendas. Foram encontradas diferenças $(p<0,05)$ entre as duas fontes minerais, onde é destacado o aumento de fósforo no solo onde a escama de peixe foi testada, cujo nível passou de 5,6 para 137 ppm, portanto a escama de peixe poderia ser usada como uma alteração para os solos ácidos, embora sua eficácia não seja igual a uma enmenda comercial, sua funcionalidade é óptima.

Palavras-chave: Enmenda, compostagem, fertilizante, solo ácido, agricultura.

\section{INTRODUCCIÓN}

La Encuesta Nacional Piscícola indica que la producción en Colombia en el 2012 alcanzó 64.021 toneladas de carne de pescado, siendo tilapia roja la especie con mayor aporte, seguida por la plateada y cachama, con 29.983, 14.400 y 9.659 toneladas respectivamente. La producción en el departamento del Meta, ocupó el segundo lugar a nivel nacional y primero en la Orinoquia, donde llegó a 7.023 toneladas, en la que la tilapia y cachama aportaron 3.731 y 3.221 toneladas respectivamente (CCl y MADR, 2012). La acuicultura es un renglón de la economía que presenta ritmos de crecimiento y rentabilidad superiores a otras actividades agropecuarias tradicionales (Guevara, 2009), y que en consecuencia, genera mejores beneficios al productor y contribuye al desarrollo global de la nación, aportando 21 mil empleos y se espera contribuir dentro del Acuerdo Nacional de Competitividad con 136 mil para el año 2020 (MADR, 2005); además se señala que, el consumo de pescado es una fuente importante de fosforo $(P)$, proteínas de alta calidad nutricional y ácidos grasos esenciales como los Omega-3 
que ejercen un efecto protector contra riesgos cardiovasculares (Perea et al., 2008).

Al mismo tiempo, la producción piscícola genera problemas ambientales en las fases de levante, engorde, transformación y fileteado, en las cuales se desvían los cursos de fuentes hídricas, contaminando el agua con las escamas e incluso vísceras que se desechan, puesto que culturalmente los residuos no se aprovechan dentro del mismo u otros procesos productivos (Velasco et al., 2012). En el caso específico de la escama, si se toma en consideración que cerca del 8\% de la masa total de cada individuo de cachama o tilapia le corresponde a este residuo (Gómez et al., 2017), se podría estimar que se descargaron, a receptores naturales, a nivel nacional y en el departamento del Meta por encima de 5.121 y 556 toneladas respectivamente en el año 2012; lo anterior es preocupante, si se tiene en cuenta que en Colombia a partir del año 2000 la acuicultura de agua dulce ha mantenido una dinámica de crecimiento continuo (Merino et al., 2013).

Por lo anterior es importante desarrollar procesos acuícolas en los que se potencien los aspectos económicos y sociales inherentes a la actividad, pero que además, se orienten a la superación de los problemas de insostenibilidad que en diferentes regiones hacen presencia (Ponce et al., 2006). De manera que, las perspectivas de la acuicultura a nivel mundial, regional y local se fundamentan en alternativas de aprovechamiento integral y racional de los recursos involucrados, y la distribución equitativa de los beneficios que brinda la producción (Brugère y Ridler, 2005). En este contexto se planteó este trabajo orientado al estudio de posibles alternativas de aprovechamiento de la escama de cachama y tilapia, con fundamento en las siguientes consideraciones: 1) La escama, que de conformidad con la información relacionada (Hurtado et al., 2013), puede contener hidroxiapatita $\left(\mathrm{Ca}_{10.6} 6 \mathrm{PO}_{4} .2 \mathrm{OH}\right)$, carbonato de calcio y una capa profunda de colágeno, constituyentes de dos fases, la mineral y orgánica por la presencia de compuestos de carbono. 2) Son ampliamente conocidas las técnicas generales de extracción de sustancias orgánicas con solventes (Valcárcel y Gómez, 1988), que además pueden permitir la separación de las fases mineral (sólida) y orgánica (en 
solución). 3) Se ha logrado aislar, extraer y caracterizar colágeno e hidroxiapatita a partir de materiales de origen animal (Rivera et al., 2003). La extracción con solventes, preferencialmente, con agua, ofrece importantes ventajas, por su bajos costos, toxicidad y peligrosidad de manejo, y relativa abundancia (Audisio et al., 2014; Gálvez, 2016); de esta manera, la fase sólida contendría el calcio y el fósforo y la solución el colágeno, de los cuales los dos primeros podrían aprovecharse en ensayos de enmiendas para suelos ácidos

\section{Cachama blanca (Piaractus brachypomus)}

La cachama blanca empezó a producirse desde 1983, con un promedio de 50 toneladas/año, y en la actualidad se obtienen entre 16.000 a 18.000 toneladas/año; su importancia comercial radica en la excelente calidad y sabor de su carne, que le da buena aceptación en el mercado; igualmente, su valor productivo depende de sus hábitos omnívoros con tendencia al consumo de frutos y semillas que le permite aceptar diferentes tipos de alimentos naturales, logrando altas tasas de conversión alimenticia (Mesa y Botero, 2007). Es una de las especies recomendables a ser explotada con estos fines comerciales, siendo un pez nativo de las regiones de la Amazonia y la Orinoquia de Sur América, de la Familia Characidae, Subfamilia Serrasalminae, que junto con la cachama negra (Colossoma macroporum) comparten igual número de cromosomas $(2 \mathrm{n}=54)$. La especie es de gran rusticidad, adaptable a condiciones limnológicas desfavorables, presenta buenos parámetros productivos con excelente palatabilidad; anatómicamente está conformada por cabeza grande, cuerpo en forma de globo, aletas dorsal, caudal y anal bien desarrolladas, además presenta un par de aletas pectorales y un par de aletas pélvicas las cuales son de color rojizo-naranja; una aleta adiposa gruesa y pequeña, característico de los miembros más primitivos de los teleósteos (Parrado, 2012).

\section{Tilapia roja (Oreochromis sp.)}

La tilapia roja es el resultado de varios cruces de esta misma especie, se originaron en África, es altamente filtradora, y son muy utilizadas en la producción 
piscícola. Para su cultivo se debe contar con poblaciones monosexo de machos por su mejor crecimiento y para evitar su pronta reproducción que traería serios problemas de superpoblación y competencia por oxígeno, espacio y alimento, y con ello la rápida propagación de enfermedades (López et al., 2007).

\section{Enmiendas utilizadas en la agricultura}

En agricultura se conoce por enmiendas aquellas sustancias que se incorporan al suelo, actuando principalmente sobre su textura, corrigiendo problemas de compactación o exceso de soltura, y modificando las reacciones químicas y/o biológicas, estimulándolas en diversas formas. Se debe mencionar que la cal actúa en parte como abono por su acción directa en la formación de tejidos vegetales, así como también interviene en la movilización de diversos elementos del suelo; dentro de las enmiendas calcáreas se encuentran el carbonato de calcio, la cal viva, la cal apagada y el yeso (Rincón et al., 2012).

- Oxido de calcio (CaO): También conocido como cal viva o cal quemada, es un polvo blanco muy difícil y desagradable de manejar. Se fabrica calcinando al horno piedra caliza lo que produce la siguiente reacción:

$$
\mathrm{CaCO}_{3}+\text { calor }\left(1000^{\circ} \mathrm{C}\right) \rightarrow \mathrm{CaO}+\mathrm{CO}_{2}
$$

Cuando se aplica en suelo acido se observan de inmediato cambios en el suelo, generando una reacción exotérmica que se debe a que, por ser un oxido se incorpora rápidamente al ponerse en contacto con el agua provocando una liberación de iones $\mathrm{OH}^{-}$(Solano, 2013).

- Hidróxido de calcio: También conocido como cal apagada (Ca.2OH), es un cristal incoloro o polvo blanco cáustico, obtenido al reaccionar óxido de calcio con agua. Puede también precipitarse mezclando una solución de cloruro de calcio con una de hidróxido de sodio (Culqui, 2017).

$$
\mathrm{CaO}+\mathrm{H}_{2} \mathrm{O} \rightarrow \mathrm{Ca} 2 \mathrm{OH}
$$


- Cal agrícola o calcita: Es el material más utilizado para encalar los suelos y contiene principalmente carbonato de calcio $\left(\mathrm{CaCO}_{3}\right)$. Se obtiene a partir de la roca caliza y roca calcárea o calcita que se muele y luego se cierne en mallas de diferente tamaño; en su forma pura contiene $40 \%$ de Ca (Molina, 1998).

- Dolomita: Se le domina así al carbonato doble de calcio con magnesio ( $\mathrm{CaCO}_{3} \cdot \mathrm{MgCO}_{3}$ ), el material puro contiene $21.6 \%$ de $\mathrm{Ca}$ y $13.1 \%$ de $\mathrm{Mg}$. Aunque la dolomita reacciona más lentamente en el suelo que la calcita, tiene la ventaja de que suministra $\mathrm{Mg}$, elemento con frecuencia deficiente en suelos ácidos (Molina, 1998).

- Yeso $\left(\mathrm{CaSO}_{4} \cdot \mathrm{2H}_{2} \mathrm{O}\right)$ : Se puede encontrar de forma común en la naturaleza debido a la precipitación del sulfato de calcio que tiene lugar en el agua del mar y suele encontrarse relacionado con la caliza, gracias a la acción del ácido sulfúrico que procede de los volcanes, a través de la siguiente reacción:

$$
\mathrm{CaCO}_{3}+\mathrm{H}_{2} \mathrm{SO}_{4} \rightarrow \mathrm{CaSO}_{4}+\mathrm{CO}_{2}+\mathrm{H}_{2} \mathrm{O}
$$

El yeso se puede obtener también de manera artificial, como un subproducto del $\mathrm{H}_{3} \mathrm{PO}_{4}$ partiendo del $\mathrm{Ca} 3\left(\mathrm{PO}_{4}\right)_{2}$, siguiendo la reacción:

$$
\mathrm{Ca}_{3}\left(\mathrm{PO}_{4}\right)_{2}+3 \mathrm{H}_{2} \mathrm{SO}_{4} \rightarrow 2 \mathrm{H}_{3} \mathrm{PO}_{4}+3 \mathrm{CaSO}_{4}
$$

Si se añade ácido sulfúrico a las rocas fosfatadas, se consigue una mezcla de yeso, con sulfato monocálcico y dicálcico; dicha mezcla es utilizada como fertilizante, dándosele el nombre de superfosfato (Martinez, 1999).

El creciente aprovechamiento en el departamento del Meta, en la Orinoquia y el país en general, en la producción de alimentos, de especies cultivadas en cautiverio como la cachama blanca (Piaractus brachypomus) y tilapia roja (Oreochromis spp), genera considerables cantidades de escama y huesos que en forma de desechos, por lo general, son descargados al ambiente circunvecino, en particular a fuentes hídricas, generando procesos de deterioro del medio natural (Borja, 2011). Al mismo tiempo la escama y los huesos contienen sustancias valiosas que podrían ser aprovechadas en actividades agrícolas de interés local, y al tiempo reducir los procesos de contaminación ambiental, dándole uso a 
materiales que hoy se desechan y no se aprovechan; de esta manera, se lograría utilizar materiales que en la actualidad, se acumulan y dispersan de manera progresiva en la región, es decir, aprovecharlos en procesos productivos comunes, aportando elementos básicos para la solución de problemas ambientales regionales vigentes y ofrecer alternativas a la producción agrícola local. Por lo anterior el objetivo de este trabajo fue evaluar alternativas de aprovechamiento de la escama de cachama blanca y tilapia roja, en procesos de producción agrícola, para el departamento del Meta y la Orinoquia colombiana.

\section{METODOLOGÍA}

Este trabajo se realizó en la granja Barcelona de la Universidad de los Llanos sede Barcelona; ubicada en el municipio de Villavicencio, kilómetro 7 vía Puerto López, localizada a $384 \mathrm{msnm}$, latitud, $04^{\circ} 0426.08$ longitud, $73^{\circ} 3450.52$ oeste. Las condiciones climáticas presentadas durante el establecimiento y evaluación del ensayo fueron: temperatura $\left(\mathrm{T}^{\circ}\right)$ maxima: $34^{\circ} \mathrm{C}$, $\mathrm{T}^{\circ}$ mínima: $15^{\circ} \mathrm{C}$, $\mathrm{T}^{\circ}$ media: $25^{\circ} \mathrm{C}$, precipitación 464.8-156.2 mm, humedad relativa 78-74\%, brillo solar 155-160 horas (IDEAM, 2016).

La extracción de las sustancias presentes en la escama de pescado se hizo por el método Soxhlet (extracción continua), por reflujo y destilación con arrastre de vapor de agua, para lo cual se colocaron los $7 \mathrm{~kg}$ de escama de pescado en un recipiente y se le agregó agua hasta que la escama quedó totalmente cubierta, posteriormente se llevó a fuego constante durante aproximadamente 6 horas, se le retiro el agua y se llevó a las bandejas de secado donde se dejaron a temperatura ambiente por 24 horas, luego se empacaron en papel aluminio y se llevaron a la mufla por 48 horas a $100^{\circ} \mathrm{C}$, una vez a temperatura ambiente se molieron obteniéndose un polvo fino, blanquecino y casi inholoro. Luego se llevaron $100 \mathrm{~g}$ de muestra al laboratorio donde le realizó análisis, encontrándose un contenido adecuado de $\mathrm{CaCO}_{3}(41.66 \%)$, puesto que está por encima de los contenidos teóricos (39.84\%) de la composición de la hidroxiapatita $\mathrm{Ca}_{10}\left(\mathrm{PO}_{4}\right)_{6}(\mathrm{OH})_{2}$, sustancia comúnmente presente en la escama y huesos de cachama y tilapia roja; el carbonato de magnesio fue $1.75 \%$. 
Se evaluaron tres tratamientos, los cuales consistieron en un testigo sin aplicación, el segundo con un $\mathrm{kg}$ de la escama de pescado y el tercero con cal dolomita en parcelas de $1 \mathrm{~m}^{2}$ en un lote de la granja de la universidad, en el cual previamente se habían tomado muestras para análisis de suelo para determinar los niveles iniciales de $\mathrm{pH}$, calcio y fosforo, y con ello poder estimar sus variaciones; 20 días después de la aplicación de las diferentes enmiendas, se realizó nuevamente el análisis de suelo.

\section{RESULTADOS Y DISCUSIÓN}

\section{Suelo antes de la aplicación de enmiendas}

La materia orgánica presente en este suelo estuvo entre 3.4 a 3.6\% (Tabla 1), es decir que se encuentra en nivel medio respecto a este contenido, aunque es apto para cualquier tipo de cultivo, además está condicionada a la cantidad, tipo y actividad microbiana del suelo, porque el mantenimiento de la fertilidad biológica sugiere inalterabilidad del ambiente, y en este aspecto son varias las ventajas, debido a que estos microorganismos participan en procesos de humificación y mineralización de la materia orgánica, fijación bilógica de nitrógeno, solubilización de componentes minerales del suelo (asociación micorrízica) y reducción de nitratos y sulfatos (Almendros et al., 1984).

El pH del suelo es acido, estando entre 4.7 y 5.1 (Tabla 1), lo cual influye de forma indirecta en procesos químicos y biológicos, disponibilidad de nutrientes y actividad microbiana. Está definido como el logaritmo inverso de la actividad de iones hidrógeno en la solución suelo. Normalmente el rango de $\mathrm{pH}$ de los suelos varía entre 3.5 a 9.0, la razón por la que no se alcanza valores extremos de 0 ó 14 se debe a que la solución del suelo es coloidal. A la mayoría de especies vegetales, les favorece $\mathrm{pH}$ entre 5.5 a 7.5 , pero cada especie y variedad tiene un rango específico donde se desarrolla mejor; normalmente entre 6.5 y 7.0 es el rango que se maneja para cultivos que están en fertirrigación. Un pH neutro o poco ácido, entre 5 y 7 favorece la disponibilidad de la mayoría de los nutrientes; los valores altos hacen menos disponible algunos nutrientes, entre ellos el fósforo, 
mientras que un $\mathrm{pH}$ muy bajo puede insolubilizar algunos nutrientes y movilizar aluminio $\left(\mathrm{Al}^{+3}\right)$, porque este catión es soluble a pH ácido, y en estas condiciones, inhibe el crecimiento de muchas especies, puesto que impide la absorción de hierro y algunos procesos metabólicos (Ramos y Zúñiga, 2008).

El contenido de calcio (Ca) estuvo entre 2 a $2.6 \mathrm{meq} / 100 \mathrm{~g}$ suelo, encontrándose este valor en un nivel más bajo que el ideal ( $5 \mathrm{meq} / 100 \mathrm{~g})$. Cuando se agrega cal (carbonato de calcio), el Ca desplaza al hidrógeno $(\mathrm{H})$ presente en la superficie de las partículas de suelo, disminuyendo su acidez; es esencial para los microorganismos que transforman los residuos de cultivos en materia orgánica, quienes liberan nutrientes y mejoran tanto la estructura como la capacidad del suelo de almacenar agua; ayuda a las bacterias del género Rhizobium a convertir el nitrógeno $(\mathrm{N})$ atmosférico en compuestos nitrogenados que las leguminosas pueden utilizar, mejora la absorción de otros nutrientes por las raíces, así como su translocación en la planta. El Ca es vital para varios procesos en la planta, incluyendo los siguientes: Conversión del nitrato $\left(\mathrm{NO}_{3}-\mathrm{N}\right)$ a formas necesarias para la formación de proteínas, activación de varios sistemas enzimáticos que controlan el crecimiento de la planta, el Ca se necesita para la formación de paredes celulares y para asegurar una división celular normal, y contribuye a mejorar la resistencia a enfermedades. El $\mathrm{Ca}$, junto con el magnesio (Mg) y el potasio $(\mathrm{K})$, ayudan a neutralizar los ácidos orgánicos presentes en la planta (Higuera et al., 2012).

Siendo el fósforo fundamental para la nutrición de las plantas, su concentración es baja, entre 4.7 a $6.1 \mathrm{ppm}$ (Tabla 1), siendo el nivel $15 \mathrm{ppm}$ y alto superior a 30 ppm, según la escala comparativa de contenido de este mineral en el suelo. Este elemento se absorbe en forma de fosfatos mono y diácidos; por su poca movilidad, y tendencia a formar componentes fosforados no disponibles para las plantas es que debe ser considerado uno de los elementos más críticos, aunque en la planta su contenido es menor que el nitrógeno, potasio, y calcio, es un factor limitante de mayor importancia que el calcio y el potasio. Este mineral es importante para la calidad y precocidad a las plantas, puesto que adelanta su maduración, a 
diferencia del nitrógeno que tiende a prolongar el crecimiento vegetativo. Se encuentra en toda la planta, y especialmente en los tejidos jóvenes y órganos de reserva; en los primeros interviene en la síntesis proteica y contribuye al desarrollo radicular, y en los últimos (semillas y tubérculos) forma parte de fosfolípidos y ácidos nucleicos; también ayuda a la acumulación de energía y combustible para todas las actividades bioquímicas de las células porque forma parte del adenosin trifosfato (ATP) (Mendoza, 1980).

Tabla 1. Análisis del suelo antes y después de los tratamientos

\begin{tabular}{cccccc}
\hline \multirow{2}{*}{ Parámetro } & \multirow{2}{*}{ Testigo* $^{*}$} & \multicolumn{2}{c}{ Cal dolomita } & \multicolumn{2}{c}{ Escamas } \\
\cline { 3 - 6 } & & Antes & Después & Antes & Después \\
\hline Materia orgánica $(\%)$ & 3.6 & 3.3 & 3.0 & 3.4 & 3.2 \\
pH & 5.1 & 4.7 & 4.9 & 6.1 & 4.8 \\
Ca $(\mathrm{ppm})$ & 2.6 & 2 & 10.65 & 2.2 & 4.35 \\
$\mathrm{P}(\mathrm{ppm})$ & 5.6 & 6.1 & 4.7 & 3.9 & 137 \\
\hline
\end{tabular}

* Al testigo no se le realizó análisis de suelos posterior asumiéndose que su composición se mantuvo igual.

\section{Análisis de suelos post aplicación de enmiendas}

La materia orgánica contiene sustancias variadas de color pardo y negruzco, que resultan de la descomposición del material vegetal, además contiene aproximadamente un $5 \%$ de nitrógeno, y tiene efecto sobre las propiedades físicas del suelo, formando agregados y dando estabilidad estructural, uniéndose a las arcillas y formando el complejo de cambio, favoreciendo la penetración del agua y su retención, disminuyendo la erosión y favoreciendo el intercambio gaseoso ( $\mathrm{Di}$ Ciocco et al., 2014). En el suelo del experimento, la materia orgánica disminuyó posterior a la aplicación tanto de cal como de escama de pescado (Figura 1).

Las sustancias húmicas se consideran la parte más recalcitrante y estable de la materia orgánica del suelo (Jindo et al., 2015). En el grupo de los ácidos húmicos están englobadas las materias que se extraen del suelo por distintos disolventes 
( $\mathrm{NaOH}, \mathrm{KOH}, \mathrm{NH}_{4} \mathrm{OH}, \mathrm{Na}_{2} \mathrm{HCO}_{3}, \mathrm{Na}_{4} \mathrm{P}_{2} \mathrm{O}_{7}, \mathrm{NaF}$, oxalato sódico y otros), que al acidificarse con ácidos minerales se precipitan de las soluciones. A pesar de la diversidad de los ácidos húmicos en los distintos suelos, turbas, restos vegetales en descomposición, éstos conservan sus principios de estructura muy semejantes; los grupos característicos de los ácidos húmicos son los carboxilos e hidroxilos fenólicos, cuyo hidrógeno es susceptible a las reacciones de sustitución. Los ácidos húmicos son ácidos polibásicos de débil disociación que tienen el punto de equivalencia cerca de un $\mathrm{pH}$ de 8-9; aparte de los grupos carboxílicos, fenólicos y alcohólicos, hay en los ácidos húmicos grupos metoxílicos $\mathrm{OCH}_{3}$, cuya cantidad en los distintos representantes es oscilante, al aplicarse las enmiendas se puede inferir que se generaron condiciones ambientales favorables para un incremento en la flora microbiana que favorecieron el incremento en las cantidades de nitrógeno asimilable y una reducción de materia orgánica (Lobartini y Orioli, 1996).

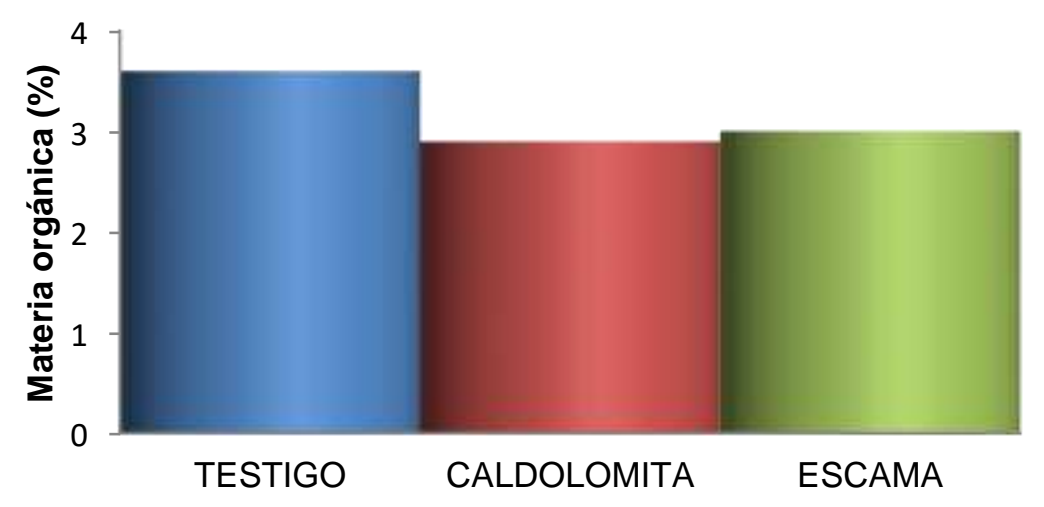

Figura 1. Comparación de la materia orgánica

Los ácidos húmicos en su composición molecular tienen iones de hidrógeno con enlaces muy débiles que en grandes cantidades pueden ocasionar la acidificación del suelo, al aplicar calcio al suelo reaccionan sobre dicho elemento, en este sentido el encalamiento influye sobre los efectos de la disponibilidad del nitrógeno, fosforo, potasio y algunos oligoelementos. Otro efecto de la aplicación de las enmiendas sobre la materia orgánica es que estimulan el metabolismo general de 
los organismos heterótrofos del suelo, con una mayor rapidez de mineralización de la materia orgánica. La mayoría de las bacterias del suelo responsables de la conversión de $\mathrm{NH}_{4}$ a $\mathrm{NO}_{3}$ requieren grandes cantidades de calcio activo, como consecuencia, el proceso de nitrificación queda favorecido. También el proceso de fijación del nitrógeno tanto simbiótico como no simbiótico, se favorece con una correcta adición de calcio, en ambos casos, los microorganismos fijadores requieren cantidades altas de calcio para el desarrollo de su actividad (Ramos et al., 2016).

Con relación al pH, no se presentaron mayores cambios en referencia a su acidez, aunque gráficamente se evidenció un aumento en el pH posterior a la aplicación de cal (Figura 2), lo anterior porque la cantidad utilizada de enmiendas (10 ton/ha) no fue suficiente, puesto que la cantidad necesaria para obtener una respuesta favorable es 20 toneladas por hectárea (Osorio, 2012).

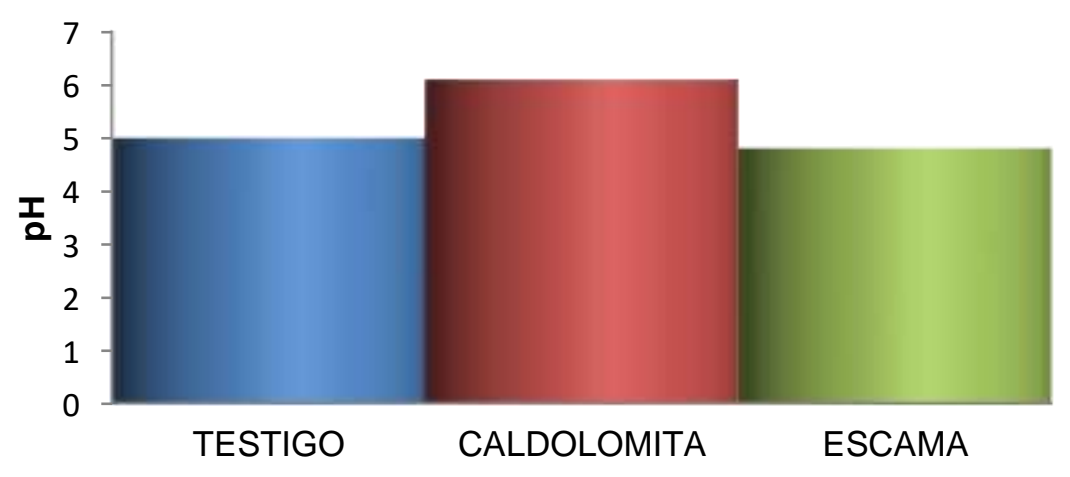

Figura 2. Respuesta de $\mathrm{pH}$ a los tratamientos

En los suelos se observó un alto incremento de este elemento, el cual paso de 3.9 ppm antes de la aplicación de enmiendas a 137 ppm con escama de pescado, y bajó de 6.1 a $3.5 \mathrm{ppm}$ con cal dolomita (Figura 3), lo cual es debido a la composición química de la escama de pescado que es rica en este elemento (190 $\mathrm{mg} / 100 \mathrm{~g}$ de fosforo). Al ir aumentando la acidez del suelo aumenta también la solubilidad de los hidroxilos libres de hierro y aluminio originándose $\mathrm{Fe}^{+3}$ y $\mathrm{Al}^{+3}$ que 
pasan a la disolución, en estas condiciones de alta acidez tanto hierro como los aluminio precipitan inmediatamente los $\mathrm{PO}_{4} \mathrm{H}_{2}$ también presentes en la disolución, originándose compuestos insolubles en los que el fosforo resulta inservible para la planta (Daza et al., 2006). Otras formas de insolubilización del fosforo que normalmente se presentan en suelos moderadamente ácidos son reacciones en las que se encuentran involucradas las arcillas donde las fijaciones y cambio de aniones se producen según el mecanismo análogo de cambio de cationes; el poder de fijación varía dependiendo del número de arcillas, puesto que aumenta o disminuye el diámetro de su partícula, indicativo de un intercambio aniónico (Mombiela y Mateo, 1982). En síntesis, el contenido de fósforo encontrado en la escama de pescado es alto y su movimiento en el suelo es bajo.



Figura 3. Variacion del contenido de fosfóro en respuesta a los tratamientos

En el suelo pre aplicación de enmiendas, el contenido de calcio era 2 y 2.2 meq/100 g lo cual es una apreciación media, posterior a la aplicación de cal dolomita subió a 10.65 ppm, mientras que con la escama de pescado llegó a 4.35 ppm (Figura 4). Cuando se aplican las dos enmiendas (cal dolomita y escama) cierta proporción tiende a solubilizarse y a cambiar a la forma bicarbonatada debido al acido carbónico del suelo; simultáneamente a este proceso disolutivo los coloides del suelo comienzan a fijar el calcio, los cuales pueden ser absorbidos directamente de la disolución del suelo o de los compuestos cálcicos aun no 
solubilizados. El posible resultado de los niveles tan altos tanto de calcio como de fosforo, puede haber sido porque los cationes disociados en la disolución junto con iones bicarbonato no han actuado como base intercambiable absorbida al coloide, y como calcio en forma de carbonato sólido, de esta forma la reserva cálcica tanto de carbonatos como de iones absorbidos proporcionan gradualmente $\mathrm{Ca}^{+2}$ a la disolución del suelo por cambio catiónico; con el tiempo, las partículas de los carbonatos cálcicos añadidos desaparecen puesto que en ese momento el complejo coloidal empieza a perder parte de calcio absorbido, al intercambiarse con los hidrogeniones proporcionados por el ácido carbónico. Los compuestos solubles del calcio son eliminados por lixiviación, y el porcentaje de saturación de bases y $\mathrm{pH}$ se reduce gradualmente (Alfaro et al., 1998).

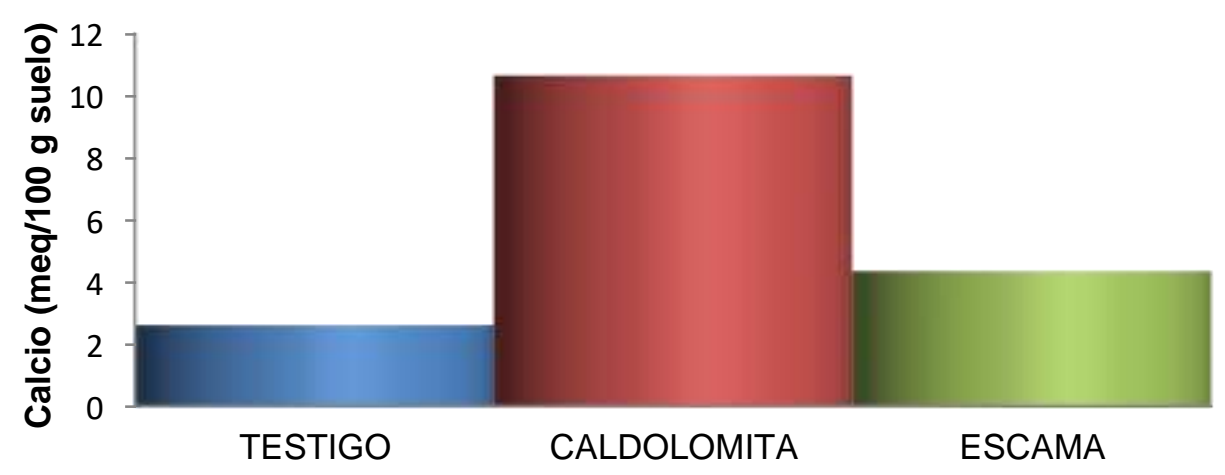

Figura 4. Cantidad de calcio en el suelo

\section{CONCLUSIÓN}

Los resultados del ensayo usando escama como enmienda en suelos con altos contenidos de aluminio, y bajos en calcio y fosforo, muestran mejorías importantes en el contenido de sus elementos, por lo tanto la escama de pescado podría ser utilizada como enmienda para suelos ácidos, aunque su efectividad no es igual a una enmienda comercial, su funcionalidad es óptima; además desde una perspectiva ecológica se presenta una alternativa para la disposición final de la 
escama, con lo cual se mejoran elementos del suelo y al mismo tiempo se evita la contaminación de aguas con la escama.

\section{RECOMENDACIONES}

Estudiar con mayor detenimiento los posibles cambios que produce la escama de pescado como enmienda enfocándose en la relación planta-suelo. Estudiar la posibilidad de hacer estudios de tallos por medio de Nitrachec y observar las cantidades de nitritos disponibles en la planta. Obtener las condiciones óptimas para desarrollar un proyecto a grande escala.

\section{REFERENCIAS BIBLIOGRÁFICAS}

1. Alfaro M., Teuber N., Dumont J.C., Medone F. Efecto del carbonato de calcio en el establecimiento y producción de gramíneas y leguminosas forrajeras en Chiloé. Agricultura Técnica (Chile). 58 (3): 173-180. 1998.

2. Almendros G., Polo A., Ibáñez J., Lobo M. Contribución al estudio de la influencia de los incendios forestales en las características de la materia orgánica del suelo. Revue d’Ecologie et de Biologie u Sol. 21 (1): 7-20. 1984.

3. Audisio S.A., Vaquero P.G., Torres P.A., Verna E.C., Ocampo L.N., Ratusnu V., Cristofolini A.L., Merkis C.I. Obtención, caracterización y almacenamiento de matriz ósea desmineralizada. Rev Med Vet (B Aires). 95 (2): 27-34. 2014.

4. Borja A. Los impactos ambientales de la acuicultura y la sostenibilidad de esta actividad. Boletín. Instituto Español de Oceanografía. 18 (1-4): 41-49. 2011.

5. Brugère $\mathrm{C}$., Ridler $\mathrm{N}$. Perspectivas de la acuicultura mundial en los próximos decenios: análisis de los pronósticos para 2030 de la producción acuícola de los principales países. Organización de las Naciones Unidas para la Agricultura y la Alimentación, Roma, Italia. 53 p. 2005.

6. CCl, Corporación Colombia Internacional y MADR, Ministerio de Agricultura y Desarrollo Rural. Encuesta Nacional Piscícola. Bogotá DC., Colombia. 20 p. 2012.

7. Culqui M.A. Influencia de la calidad de las calizas para la producción de cal viva en la calera la Conga del caserío de Sogorón Alto Distrito de la Encañada, Cajamarca 2017, Ingeniero de Minas. Facultad de Ingeniería, Universidad Privada del Norte, Cajamarca, Perú. 27 p. 2017.

8. Daza M.C., Álvarez J.G., Rojas L.A. Efecto de materiales orgánicos e inorgánicos sobre las fracciones de fósforo de un Oxisol de los Llanos Orientales colombianos. Agronomía Colombiana. 24 (2): 326-333. 2006.

9. Di Ciocco C.A., Sandler R.V., Falco L.B., Coviella C.E. Actividad microbiológica de un suelo sometido a distintos usos y su relación con variables físico-químicas. Revista de la Facultad de Ciencias Agrarias. Universidad Nacional de Cuyo. 46 (1): 73-58. 2014. 
10. Gálvez D.C. Obtención y caracterización de hidroxiapatita dopada con nanopartículas de $\mathrm{ZnO}$ con potenciales aplicaciones biomédicas, Magister en Física. Facultad de Ciencias Exactas y Naturales, Departamento de Física y Química, Universidad Nacional de Colombia-Sede Manizales, Manizales, Colombia. 58 p. 2016.

11. Gómez A.G., Ramos R., Salazar H.T. Aprovechamiento de las escamas de la industria acuícola en el departamento del Huila, Colombia. Producción + Limpia. 11 (2): 102-110. 2017.

12. Guevara C.E. Estudio de factibilidad y puesta en marcha de una empresa productora y comercializadora de mojarra roja, Ingeniero Industrial. Escuela de Ingeniería y Administración. Facultad de Ingeniería Industrial, Universidad Pontificia Bolivariana Seccional Bucaramanga, Bucaramanga. 136 p. 2009.

13. Higuera C.H., Gómez J.C., Pardo Ó.E. Caracterización de un suelo arcilloso tratado con hidróxido de calcio. Revista Facultad de Ingeniería. 21 (32): 21-40. 2012.

14. Hurtado V.L., Herrera Y.M., Gómez D.A. Efecto del uso de la escama de pescado en la alimentación de codornices sobre la calidad del huevo. CITECSA. 4 (6): 59-69. 2013.

15. IDEAM, Instituto de Hidrología, Meteorología y Estudios Ambientales. Tiempo y clima. 2016. Recuperado 16 Diciembre 2016. Disponible En: http://www.ideam.gov.co/

16. Jindo K., Chocano C., Melgares de Aguilar J., González D., Hernández T., García C. Efecto de la aplicación de compost en las fracciones de carbono y humificación del suelo en una finca ecológica frutícola. En: V Congreso Latinoamericano de Agroecología-SOCLA (7 al 9 de octubre de 2015, La Plata). 2015.

17. Lobartini J., Orioli G. Las sustancias húmicas y la nutrición vegetal. Revista de la Facultad de Agronomía, La Plata. 101 (2): 201-209. 1996.

18. López C.A., Carvajal D.L., Botero M.C. Masculinización de tilapia roja (Oreochromis spp) por inmersión utilizando 17 alfa-metiltestosterona. Revista Colombiana de Ciencias Pecuarias. 20 (3): 318-326. 2007.

19. MADR, Ministerio de Agricultura y Desarrollo Rural. Acuerdo de competitividad de la cadena de la piscicultura en Colombia. Bogotá DC., Colombia. 43 p. 2005.

20. Martinez M.C. Estudio económico-técnico de la explotación de sulfato sódico y diseño de la mina de yeso en Belorado-Burgos-España, Ingeniero de Minas. Facultad de Ingeniería en Ciencias de la Tierra, Escuela Superior Politécnica del Litoral, Guayaquil, Ecuador. 1999.

21. Mendoza R.E. Adsorción de fósforo en el suelo y su relación con la producción de dos especies forrajeras. Revista de la Facultad de Agronomía. 1 (2): 19-30. 1980.

22. Merino M.C., Bonilla S.P., Bages F., Diagnóstico del estado de la acuicultura en Colombia. Plan Nacional de Desarrollo de la Acuicultura Sostenible en Colombia AUNAP-FAO. Ministerio de Agricultura y Desarrollo Rural, Bogotá, Colombia. 160 p. 2013. 
23. Mesa M.N., Botero M.C. La cachama blanca (Piaractus brachypomus), una especie potencial para el mejoramiento genético. Revista Colombiana de Ciencias Pecuarias. 20 (1): 79-86. 2007.

24. Molina E. Acidez de suelo y encalado, 1998.

25. Mombiela F.A., Mateo M.E. Respuesta a seis dosis de $P$ y de cal en el establecimiento de praderas permanentes en dos tipos de suelos gallegos a monte. Pastos. 12 (1): 187-201. 1982.

26. Osorio N.W. Como determinar los requerimientos de cal del suelo. Manejo integral del suelo y nutrición vegetal. 1 (5): 1-6. 2012.

27. Parrado Y.A. Historia de la acuicultura en Colombia. AquaTIC. (37): 60-77. 2012.

28. Perea A., Gómez E., Mayorga Y., Triana C.Y. Caracterización nutricional de pescados de producción y consumo regional en Bucaramanga, Colombia. Archivos latinoamericanos de nutrición. 58 (1): 91-97. 2008.

29. Ponce J.T., Romero O., Castillo S., Arteaga P., Ulloa M., González R., Febrero I., Esparza H. El desarrollo sostenible de la acuicultura en América Latina. Revista Electrónica de Veterinaria REDVET. 7 (07): 1-16. 2006.

30. Ramos C., Obrador J., García E., Pérez J., Carrillo E. Efecto del encalado en la materia orgánica del suelo en un sistema agroforestal. Agroproductividad. 9 (12): 28-33. 2016.

31. Ramos E., Zúñiga D. Efecto de la humedad, temperatura y $\mathrm{pH}$ del suelo en la actividad microbiana a nivel de laboratorio. Ecología aplicada. 7 (1-2): 123130. 2008.

32. Rincón Á., Baquero J., Hernando F. Manejo de la nutrición mineral en sistemas ganaderos de los Llanos Orientales de Colombia. CORPOICA (Corporación Colombiana de Investigación Agropecuaria, Villavicencio, Meta, Colombia. 164 p. 2012.

33. Rivera J.A., Riaño C.H., Monsalve P.A., Osorio A. Injertos óseos-Nueva alternativa. Fase I. Extracción de proteínas morfogenéticas parcialmente purificadas de hueso bovino. Revista Colombiana de Ciencias Pecuarias. 16 (2): 139-146. 2003.

34. Solano M. Evaluación de dosis y fuentes de enmiendas en la fertilidad del suelo y el crecimiento de plantas de banano in vitro en condiciones de vivero, Licenciatura en Ingeniería Agronómica con énfasis en Fitotecnia. Facultad de Ciencias Agroalimentarias, Universidad de Costa Rica, Costa Rica. 64 p. 2013.

35. Valcárcel C.M., Gómez H.A. Técnicas analíticas de separación. Editorial Reverté S.A, Barcelona, España. 779 p. 1988.

36. Velasco P., Calvario O., Pulido G., Acevedo O., Castro J., Román A. Problemática ambiental de la actividad piscícola en el estado de Hidalgo, México. Ingeniería. 16 (3): 165-174. 2012. 